\title{
Phase Retrieval by Combined Bragg and Fresnel X-Ray Diffraction Imaging
}

\author{
P. Rejmánková-Pernot, P. Cloetens,* J. Baruchel, and J.-P. Guigay ${ }^{\dagger}$ \\ European Synchrotron Radiation Facility, BP 220, F-38043 Grenoble, France \\ P. Moretti \\ LPCML, Université Claude Bernard-Lyon I, F-69622 Villeurbanne, France
}

(Received 26 March 1998)

\begin{abstract}
Contrast due to Fresnel diffraction is observed on Bragg diffraction images, obtained with a spatially coherent x-ray beam, of ferroelectric domains inside a periodically poled lithium niobate crystal. It originates mainly from the phase shift between the structure factors of the adjacent domains. This allows the direct determination of the relative phase of the pairs of structure factors involved. [S0031-9007(98)07399-2]

PACS numbers: 61.72.Ff, 61.72.Mm, 77.84.Dy
\end{abstract}

When two parts of a coherent wave front suffer a relative phase shift, an interference pattern is produced, and the phase difference can be extracted from the fringes. An especially simple case arises when the phase distribution across the wave front is an almost ideal straight step, as when a neutron wave front is divided at a Bloch wall separating two domains with opposite magnetization [1]. The phase shift, which can be described in terms of the opposite precession directions of the neutron spin, then shows up vividly in the resulting Fresnel diffraction pattern. Free space propagation, i.e., Fresnel diffraction, makes phase sensitive radiography possible and instrumentally simple thanks to the spatial coherence of third generation synchrotron radiation beams [2,3]. In the present work, Fresnel and Bragg diffraction are simultaneously used to visualize ferroelectric domains within a periodically poled lithium niobate crystal. The wave front of the Braggdiffracted $\mathrm{x}$-ray beam is split by the phase difference between the structure factors of adjacent domains. This shift is measured through quantitative analysis of the image contrast as a function of propagation distance.

Lithium niobate can achieve optical second-harmonic generation with enhanced efficiency when a periodic reversal of the sign of the nonlinear optical coefficient $d_{33}$ occurs in the phase matching period [4]. This periodically inverted domain structure can be produced by applying an external electric field using patterned electrodes [5-7]. Various methods revealing ferroelectric domains in $\mathrm{LiNbO}_{3}$ were reported: chemical etching [8], electron microscopy $[9,10]$, pyroelectrically induced electron emission [11], and x-ray Bragg-diffraction imaging ("topography") [12-16].

The experiments were carried out at the ID11 and ID19 wiggler beam lines of the European Synchrotron Radiation Facility (ESRF). These beam lines exhibit the same vertical $s_{v}(\approx 40 \mu \mathrm{m})$ and horizontal $s_{h}(\approx 200 \mu \mathrm{m})$ FWHM source sizes, but different source-to-sample distances $L: 40 \mathrm{~m}$ for ID11 and $145 \mathrm{~m}$ for ID19. This implies, for a typical wavelength $\lambda=0.7 \AA$, that the vertical transverse coherence length $\left(l_{c}=\lambda / 2 \alpha\right.$, where the divergence $\alpha$ is ideally given by the angular source size $s / L)$ is $\approx 35 \mu \mathrm{m}$ for ID11 and $130 \mu \mathrm{m}$ for ID19. The simple setup designed to perform white beam section topography is schematically represented in Fig. 1(a). Several reflecting planes fulfill the Bragg condition of diffraction for different wavelengths, and a Laue pattern is produced, where each spot is a section topograph. This technique is usually used to reveal small distortions within nearly perfect crystals. However, it allows one, when using a coherent beam, to observe phase shifts within the diffracted beam. The sample-to-detector distance $D_{d}$ determines the defocusing distance $D=D_{d} L /\left(L+D_{d}\right)$ [17]. The images were recorded on Kodak Industrex SR films with exposure times ranging from one second to a few minutes. The periodically poled sample was produced by bulk electric-field poling [6] using a $Z$-cut wafer from a congruent $\mathrm{LiNbO}_{3}$ crystal supplied by Crystal Technology. The domain reversal, with a period of $23 \mu \mathrm{m}$, was performed across the whole thickness of $0.5 \mathrm{~mm}$ of the crystal.

Figures 1(b)-1(e) show a set of section topographs obtained using the 274 reflection for different defocusing distances $D$. This procedure is used because only "standard" topographic images [18] are observed when the detector is close to the crystal, whereas phase-related contrast can build up when $D$ is increased. The slits were perpendicular to the domain walls, as shown on Fig. 1(a), the diffraction plane was approximately vertical. Only very faint contrast of inverted domains is found in Fig. 1(b), recorded at $D=0.11 \mathrm{~m}$. Stronger contrast appears over the whole section topograph of Fig. 1(c), corresponding to $D=0.51 \mathrm{~m}$. This contrast consists of parallel lines periodically spaced, with a period equal to the period of poling. The contrast increases with $D$ [Figs. 1(d) and 1(e)].

The geometrical resolution $\left(D_{d} s / L\right)$ and the transverse coherence are better when Bragg diffraction occurs in the horizontal plane and the domain walls are horizontal. Figure 2 shows the $0 \overline{3} 18$ section topographs recorded 

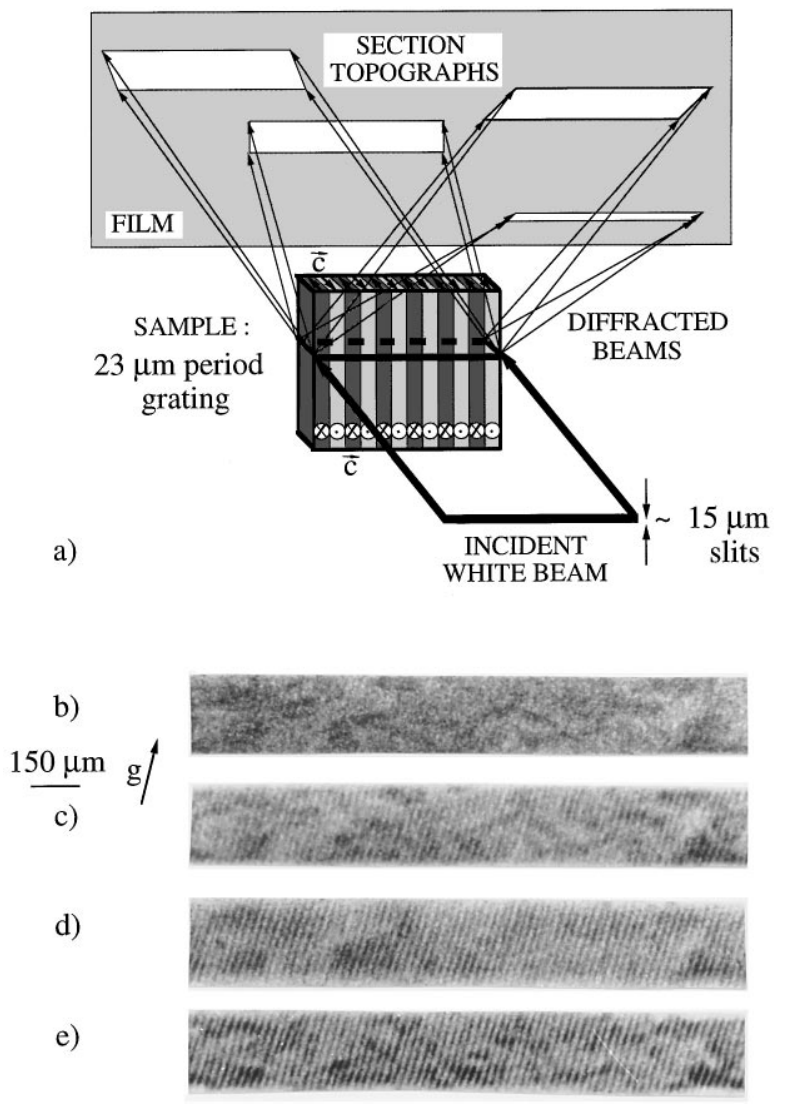

FIG. 1. (a) Principle of white beam section topography: the defects present in a single crystal can be observed within the Laue spots, and localized with respect to the crystal surfaces. (b)-(e): Section topographs using the 274 reflection, $\lambda=$ $0.24 \AA$, recorded at different defocusing distances $D$; (b) $D=$ $0.11 \mathrm{~m}$, (c) $D=0.51 \mathrm{~m}$, (d) $D=1.11 \mathrm{~m}$, (e) $D=1.67 \mathrm{~m}$; $\mathbf{g}$ is the projection of the diffraction vector on the detector.

for different defocusing distances using this horizontal diffraction plane geometry. The images observed at large distances in Figs. 2(d) and 2(e) consist of periodic lines, similar to Figs. 1(d), 1(e). But the section topographs obtained at smaller distances [Figs. 2(b), 2(c)] display finer features: Additional black and white lines can be observed between the main ones. Some image features cross the edges of the section topograph. They correspond to an asterism effect [19] associated with misorientations present in the neighborhood of the surface, which produce an elongation of the images of both domains along the diffraction vector.

It is obvious that the images vary strongly as a function of the defocusing distance. This type of behavior may arise from (1) different orientations of the reflecting planes ("misorientation" contrast). However, this is not the main origin of the observed features, for at least three reasons: (i) The dependency of contrast on the propagation distance does not correspond to the expected behavior (i.e., periodic alternation of black, grey, and white lines) of misorientation contrast between adjacent domain as a function of the sample-to-film distance. (ii) The observed

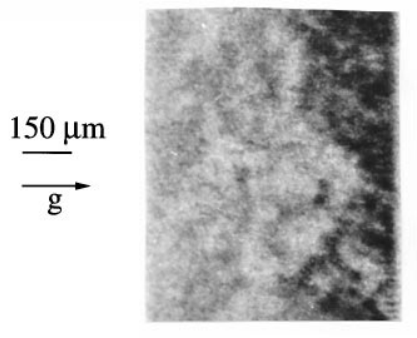

a)

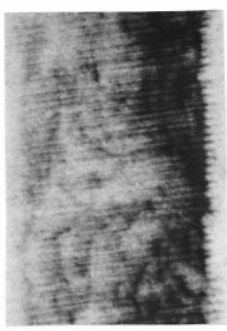

c)

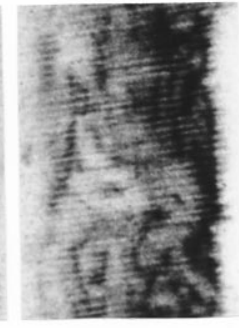

d)

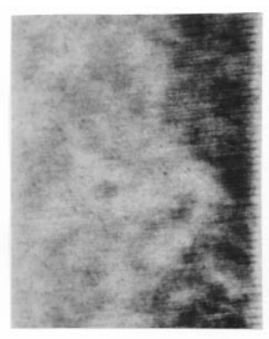

b)

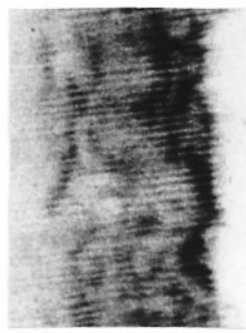

e)
FIG. 2. Section topographs using the $0 \overline{3} 18$ reflection at $\lambda=$ $0.43 \AA$ recorded at different defocusing distances $D$ : (a) $D=$ $0.12 \mathrm{~m}$, (b) $D=0.6 \mathrm{~m}$, (c) $D=1.95 \mathrm{~m}$, (d) $D=3.5 \mathrm{~m}$, (e) $D=5 \mathrm{~m}$.

contrast strongly depends on the incident white beam spectrum, which can be modified by varying the gap of the wiggler source. The relative contribution of the reflection and its harmonics is altered, resulting in a dramatic change, or even the vanishing, of the observed contrast. This is in contradiction with the expected behavior of misorientation contrast, which is not sensitive to the spectrum variation. (iii) The diffraction plane is nearly parallel to the domain walls. The Bragg angle $\theta$ is therefore modified by the misorientations multiplied by $\sin \theta(\approx 0.1)$, and one should observe a much larger effect when working with the diffraction plane perpendicular to the domain walls. This is exactly opposite to the observations. The misorientation can, nevertheless, play a role in the neighborhood of the surface, or if an external parameter (electric field [15], temperature) is applied.

The strong variance of images as a function of the defocusing distance may also arise from (2) different structure factors ("structure factor" contrast). Reflecting planes $h k l$ in domain I correspond to planes $-h,-k,-l$ in the inverted structure of domain II. It is important to note that the relative difference of the moduli $\left|F_{h k l}\right|$ and $\left|F_{-h,-k,-l}\right|$, calculated using the Fhkl software [20], with dispersion corrections, of course, taken into account, is less than $1 \%$ and therefore cannot explain the observed contrast. $F_{h k l}$ and $F_{-h,-k,-l}$ differ however substantially in their phases $\varphi_{\mathrm{I}}$ and $\varphi_{\mathrm{II}}$. The waves diffracted by adjacent domains exhibit, when they leave the crystal, a phase-shift $\Delta \varphi=\left|\varphi_{\mathrm{I}}-\varphi_{\mathrm{II}}\right|$, which is zero for simple transmission, i.e., in the absence of Bragg diffraction. Interference between parts of the wave front diffracted by different domains then occurs through free space propagation, well 
described as Fresnel diffraction. As in the case of phase radiography, this turns the phase modulation into an amplitude and a corresponding intensity modulation. This requires the transverse coherence length $l_{c}$ of the beam to be larger than the first Fresnel zone diameter $2 \sqrt{\lambda D}$. This zone is the region of the object mainly contributing to the intensity in a point of the image.

For a periodic object ("Talbot effect" [21,22]) the Fresnel diffraction pattern is periodic both in the direction of the object periodicity (period $a$ ) and in the propagation direction (period $D_{T}=2 a^{2} / \lambda$ ). In the case of a phase object, no contrast is expected immediately behind the object. It increases up to a maximum and vanishes again at a distance $D_{T} / 2$. At a distance $D_{T} / 4$ a simple relationship exists between the phase object and the intensity $I(x)$ on the image, $I(x)=1+\sin [\varphi(x)-\varphi(x+a / 2)]$ [22]. This leads in our case to a periodic intensity profile similar to the domain pattern itself, with a visibility $\left[\left(I_{\max }-I_{\min }\right) / I_{\text {mean }}\right]$ given by $2 \sin (\Delta \varphi)$. The Talbot effect for hard $\mathrm{x}$ rays was recently demonstrated in simple transmission geometry on a pure phase grating [23].

A quantitative approach giving access to the phase shift and the angular divergence of the wave exiting the crystal is shown on Fig. 3. In the case of a periodic object, the Fourier coefficients $I_{m}(D)$ of the intensity profile recorded at distance $D$ can be written as [24]:

$$
I_{m}(D)=I_{m, \operatorname{coh}}(D) \gamma(\lambda D m / a) R(m / a) .
$$

The coefficients $I_{m, \mathrm{coh}}(D)$ correspond to illumination by an ideally coherent plane wave, and they were numerically calculated within the Fresnel diffraction approximation. The periodic phase modulation used is shown in the inset of Fig. 3. It is characterized by a phase shift $\Delta \varphi$ and a linear transition of width $w$ between the two phase levels. The term $\gamma(\lambda D m / a)$ is the degree of coherence in the object plane between two points separated by the distance $(\lambda D m / a)$. By the Zernike-Van Cittert theorem [17] it can be related to the angular beam pro-

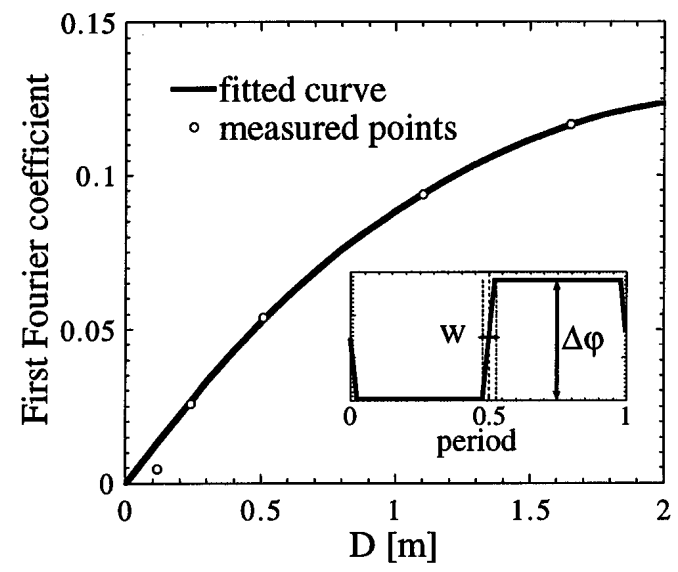

FIG. 3. Amplitude of the first Fourier coefficient as a function of defocusing distance for the 274 reflection at $\lambda=0.24 \AA$. The fitted curve corresponds to Eq. (1) and the phase modulation shown in the inset. file, yielding $\gamma(\lambda D m / a)=\exp \left[-(\alpha \pi D m / 2 a)^{2} / \ln 2\right]$ for an angular profile of Gaussian shape and width $\alpha$. The resolution of the detector is taken into account by its modulation transfer function $R(m / a)$ for the spatial frequency $m / a$.

Expression (1) was used to fit our experimental data as a function of $D$ for $m=1$, the higher-order Fourier coefficients being too weak for interpretation. The estimated value of $80^{\circ}$ for $\Delta \varphi$ is consistent with the phase shift of $70^{\circ}$ calculated on the basis of the accepted structure of lithium niobate [25]. The transition width $w$ is small (5\% of the period) and is attributed to the diffraction geometry and the finite extension, about $20 \mu \mathrm{m}$, of the incident beam in its narrow direction. The beam divergence resulting from the fit, $3.6 \mu \mathrm{rad}$, is larger than the angular source size, and results from the crystal quality of the sample coupled with the Darwin width of this reflection, i.e., the width of the intrinsic rocking curve for a perfect crystal.

The simulated intensity distributions shown in Figs. 4(a), 4(b) are in good qualitative agreement with the experimental images of Figs. 1 and 2. They are based on the phase changes associated with the domain structure, and do not take into account the defects present in the real crystal. A fine structure can be seen both in Figs. 2(b), 2(c) and on the corresponding simulations. The agreement is better in some regions of the sample, indicating that the domain structure is not completely periodic. In a later stage, it should be possible to reconstruct the x-ray amplitude distribution at the exit of the crystal and the corresponding domain distribution from the experimental images using an adapted algorithm [26]. In the case of Fig. 2, the crystal quality has a smaller effect on the divergence, because the Darwin width of the used reflections is much larger than the corresponding one for Fig. 1. Therefore the ideal divergence of $0.3 \mu \mathrm{rad}$, resulting from the angular source size, was used in Fig. 4(b).

The simulations neglect the contribution of the higher harmonics, e.g., reflection $2 h, 2 k, 2 l$ corresponding to wavelength $\lambda / 2$, and assume the diffraction vector is exactly parallel to the domain walls. This simplification of the diffraction geometry eliminates the possibility of multiple diffraction in different domains. Domain boundary contrast attributed to the phase difference between structure factors can build up within the crystal and it was formerly interpreted on the analog of the contrast of a stacking fault in the dynamical theory of x-ray diffraction [27]. In the present case, a weak "dynamical contrast" is observed only close to the sample on reflections with larger Darwin widths, and it is neglected.

The atomic displacement of the niobium ion, $0.54 \AA$ along the $c$ axis [25], is the main contribution to the phase shift between the structure factors of neighboring domains. However, a weak contrast is observed on most of the $h k 0$ reflections. The anharmonic thermal vibrations [25] are a possible origin of the phase shift for these reflections. 


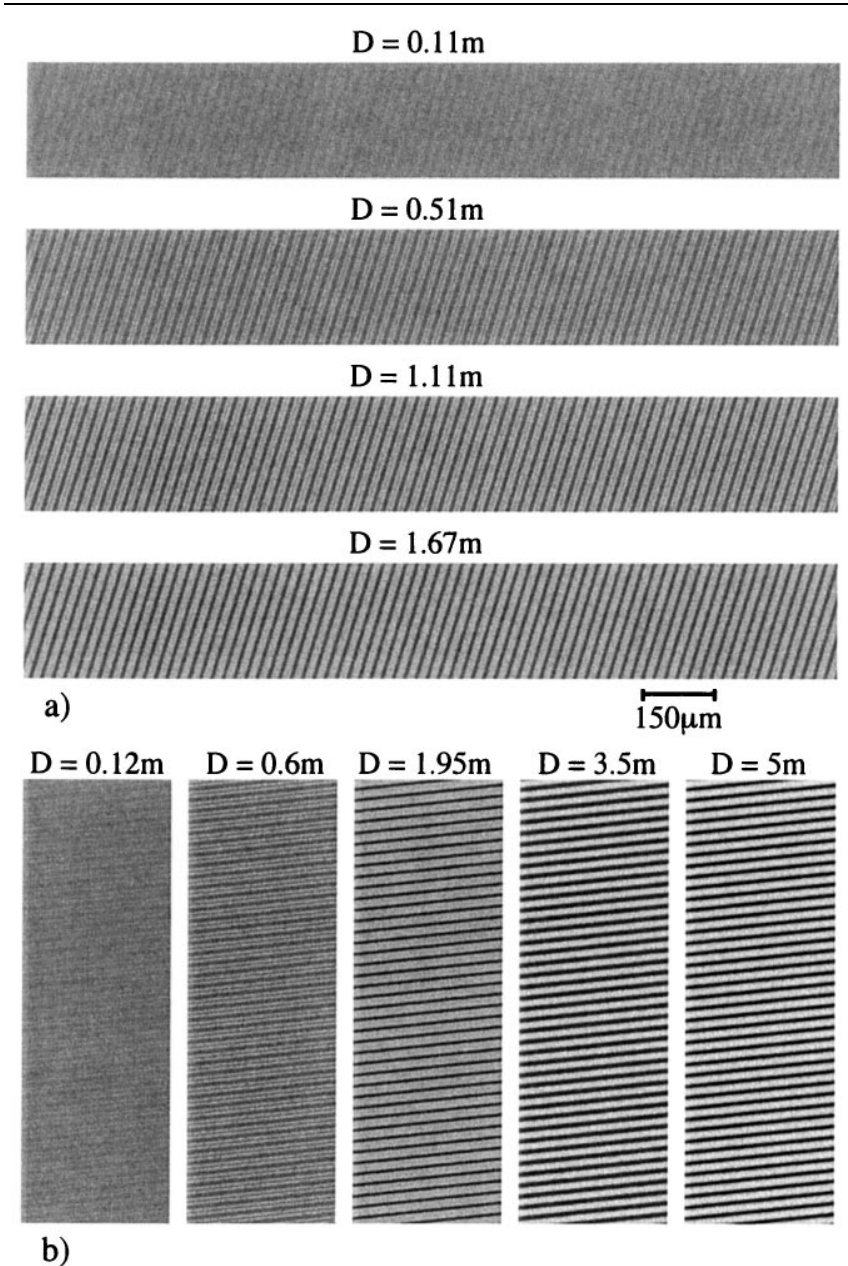

b)

FIG. 4. Simulated intensity distributions for different experimental distances $D$; (a) the 274 reflection, $\lambda=0.24 \AA$, $\Delta \varphi=80^{\circ}, \quad w=0.05$, beam divergence $\alpha=3.6 \mu \mathrm{rad}$, (b) the $0 \overline{3} 18$ reflection, $\lambda=0.43 \AA, \Delta \varphi=86^{\circ}, w=0$, $\alpha=0.3 \mu \mathrm{rad}$. The detector resolution and the object period are 4 and $23 \mu \mathrm{m}$, respectively.

A low temperature experiment we performed is not in contradiction with this possibility.

The coherence of the x-ray beams available at the ESRF thus allows one to visualize, in a simple way, the distribution of inverted ferroelectric domains within the volume of periodically poled crystals produced for nonlinear optics applications. Bragg diffraction introduces a phase shift between the waves diffracted in adjacent domains due to the phase difference between their structure factors. Fresnel diffraction and its special manifestation for periodic objects, the Talbot effect, determine the intensity variations after free space propagation. Through the measurement of the observed contrast as a function of propagation distance, the phase difference between the structure factors of reflections $h k l$ and $-h,-k,-l$ can be directly obtained. This approach is a new way to investigate any crystal feature which introduces a distortion of the phase of the Bragg diffracted wave. In particular, it allows one to determine precisely the atomic positions in twinned noncentrosym- metric crystals, and their variations as a function of an external parameter.

We are grateful to M. Arbore, M. Fejer, and G. Foulon from Stanford University for poling and supplying the periodically poled $\mathrm{LiNbO}_{3}$ crystal. P.C. is indebted to the FWO-Vlaanderen for financial support.

*Also at EMAT, University of Antwerp (RUCA), B-2020 Antwerp, Belgium.

${ }^{\dagger}$ Also at Laboratoire Louis Néel, CNRS, B.P. 166, F-38042 Grenoble, France.

[1] A. G. Klein and G. I. Opat, Phys. Rev. Lett. 37, 238 (1976).

[2] A. Snigirev et al., Rev. Sci. Instrum. 66, 5486 (1995).

[3] P. Cloetens et al., J. Phys. D 29, 133 (1996).

[4] J. A. Armstrong, N. Bloemberger, J. Ducuing, and P.S. Pershan, Phys. Rev. 127, 1918 (1962).

[5] M. Yamada, N. Nada, M. Saitoh, and K. Watanabe, Appl. Phys. Lett. 62, 435 (1993).

[6] L.E. Myers et al., J. Opt. Soc. Am. B 12, 2102 (1995).

[7] Shi-ning Zhu et al., J. Phys. D 28, 76 (1996).

[8] K. Nassau, H. J. Levinstein, and G. M. Loiacono, Appl. Phys. Lett. 6, 228 (1965).

[9] B. J. Wicks and M. H. Lewis, Phys. Status. Solidi 26, 571 (1968).

[10] M. Houe and P. D. Townsend, Appl. Phys. Lett. 66, 2667 (1995).

[11] V.D. Kugel, G. Roseman, and D. Shur, J. Phys. D 28, 2360 (1995).

[12] C. A. Wallace, J. Appl. Crystallogr. 3, 546 (1970).

[13] T. Vreeland, Jr. and V.S. Speriosu, in Application of X-ray Topographic Methods to Materials Science, edited by S. Weissmann, F. Balibar, and J.-F. Petroff (Plenum Press, New York and London, 1984), p. 501.

[14] Z.W. Hu, P. A. Thomas, and J. Webjörn, J. Appl. Crystallogr. 29, 279 (1996).

[15] P. Rejmánková et al., J. Appl. Crystallogr. 31, 106 (1998).

[16] Z.W. Hu et al., Nature (London) 392, 690 (1998).

[17] M. Born and E. Wolf, Principle of Optics (Pergamon Press, Oxford, New York, 1980), 6th ed., p. 382.

[18] J. Baruchel, in Neutron and Synchrotron Radiation for Condensed Matter Studies, edited by J. Baruchel, J.L. Hodeau, M. S. Lehmann, J. R. Regnard, and C. Schlenker (Springer-Verlag, Berlin, 1993), Vol. I, p. 399.

[19] A. Guinier, Théorie et Technique de la Radiocristallographie (Dunod, Paris, 1964), p. 327.

[20] A. Soyer, J. Appl. Crystallogr. 28, 244 (1995).

[21] H. F. Talbot, Philos. Mag. 9, 401 (1836).

[22] J.-P. Guigay, Opt. Acta 18, 677 (1971).

[23] P. Cloetens et al., Opt. Lett. 22, 1059 (1997).

[24] J.-P. Guigay, Optik 49, 121 (1977).

[25] S.C. Abrahams and P. Marsh, Acta Crystallogr. Sect. B 42, 61 (1986).

[26] W. Coene, G. Janssen, J. Op de Beeck, and D. Van Dyck, Phys. Rev. Lett. 69, 3743 (1992).

[27] K. Takahashi and M. Takagi, J. Phys. Soc. Jpn. 44, 1266 (1978). 